\title{
Ultrashort $x$-ray pulse generation by nonlinear Thomson scattering of a relativistic electron with an intense circularly polarized laser pulse
}

\author{
F. Liu* and O. Willi \\ Institut für Laser- und Plasmaphysik, Heinrich-Heine-Universität Düsseldorf, Universitätsstraße, 1, 40225 Düsseldorf, Germany
}

(Received 4 November 2011; published 17 July 2012)

\begin{abstract}
The nonlinear Thomson scattering of a relativistic electron with an intense laser pulse is calculated numerically. The results show that an ultrashort $\mathrm{x}$-ray pulse can be generated by an electron with an initial energy of $5 \mathrm{MeV}$ propagating across a circularly polarized laser pulse with a duration of 8 femtosecond and an intensity of about $1.1 \times 10^{21} \mathrm{~W} / \mathrm{cm}^{2}$, when the detection direction is perpendicular to the propagation directions of both the electron and the laser beam. The optimal values of the carrier-envelop phase and the intensity of the laser pulse for the generation of a single ultrashort x-ray pulse are obtained and verified by our calculations of the radiation characteristics.
\end{abstract}

DOI: 10.1103/PhysRevSTAB.15.070702

PACS numbers: 41.60.Ap, 41.75.Jv, 42.65.Ky, 42.65.Re

\section{INTRODUCTION}

Thomson scattering is a well-known phenomenon in laser-plasma interactions. In the low laser intensity limit, the motion of an electron initially at rest is nonrelativistic and the impact of the magnetic field can be neglected. The oscillation of the electron in the electric field of the laser pulse results in the radiation at the same frequency as the incident laser pulse, which was first explained by Thomson [1]. With the advances of the ultrashort intense laser technology based on the principle of chirped pulse amplification [2], compact tabletop laser systems delivering laser pulses with multiterawatt peak power and a few tens of femtoseconds pulse duration are available worldwide. The laser intensity can easily go beyond $10^{19} \mathrm{~W} / \mathrm{cm}^{2}$ when it is focused. The electron motion in such an intense field becomes relativistic with a quivering velocity close to the speed of light, so that the force exerted on an electron by the magnetic field of the laser pulse is comparable to that by the electric field and cannot be ignored anymore. The relativistic nonlinear motion of an electron is predicted to be able to generate harmonics of the laser light [3], a process known as nonlinear Thomson scattering which has been widely studied by both theoretical and experimental methods.

The dynamics and radiation of an electron initially at rest in an arbitrarily intense, elliptically polarized plane electromagnetic wave were discussed in detail by Sarachik and Schappert [4]. The results were generalized to an electron initially moving with an arbitrary velocity by Salamin and Faisal [5]. A theory describing the nonlinear Thomson scattering of continuous intense plane waves

\footnotetext{
*Feng.Liu@uni-duesseldorf.de
}

Published by the American Physical Society under the terms of the Creative Commons Attribution 3.0 License. Further distribution of this work must maintain attribution to the author(s) and the published article's title, journal citation, and DOI. with electron beams and plasmas was presented by Esarey et al. [6]. The electron orbit and momentum were solved analytically for both linearly and circularly polarized laser beams. The explicit expressions for the angular and spectral distribution of the radiation were obtained. The spatial and spectral characteristics of the nonlinear Thomson scattering were also analyzed theoretically and numerically by many other authors [7-12].

The first experimental confirmation of the predictions of nonlinear Thomson scattering by the interactions of intense laser pulses with free electrons was demonstrated by Chen et al. [13]. It was shown that the second and third harmonic radiation from the underdense plasma had the unique angular patterns of nonlinear Thomson scattering predicted by theory. Efforts in detection of the radiation from nonlinear Thomson scattering continued because the process is an alternative method to generate high order harmonics reaching the x-ray regime and will not saturate when the laser intensity increases as the harmonics generated by bound electrons in atomic gas. High order harmonics well collimated in the laser propagation direction from nonlinear Thomson scattering were observed in the extreme ultraviolet radiation spectral range [14]. The radiated photon energy was extended to the $\mathrm{x}$-ray regime later [15].

All of the above-mentioned publications focus on the spectral and angular distributions of the radiation from the nonlinear Thomson scattering process. The temporal characteristics were first investigated by Lee et al. [16]. By numerical calculations, it was shown that an attosecond pulse train with photon energies ranging from 100 to $600 \mathrm{eV}$ can be generated by an electron in the laser field with a duration of 20 femtosecond and an intensity of $10^{20} \mathrm{~W} / \mathrm{cm}^{2}$.

The generation of ultrashort electromagnetic pulses, especially the isolated single subfemtosecond pulse, has drawn great interest due to the potential applications in the measurement and control of ultrafast processes [17]. 
A single attosecond pulse can be produced by $90^{\circ}$ linear Thomson scattering of a relativistic electron with a singlecycle laser pulse with controlled carrier-envelop phase [18]. In the field of a tightly focused intense laser pulse, the electron is quickly scattered away from the focused regime. The radiation emitted at the focus is much stronger than that at any other position results in the emission of a single attosecond pulse [19]. Even an isolated single $x$-ray pulse in the zeptosecond time scale can be obtained by nonlinear Thomson backscattering of a $250 \mathrm{MeV}$ electron with a tightly focused laser pulse [20]. Recently, it was proposed that a single attosecond $\mathrm{x}$-ray pulse can be generated by two counterpropagating circularly polarized laser pulses with durations of about 1.5 laser cycles [21]. However, in all of these proposed schemes, intense laser pulses with durations very close to only a single laser cycle or focused beam waists of a few wavelengths are necessary, which are quite difficult to be realized experimentally.

In this paper, we show that a single ultrashort $\mathrm{x}$-ray pulse can be generated by an electron with an initial energy of $5 \mathrm{MeV}$ propagating across a circularly polarized laser pulse with a pulse duration of 8 femtosecond and an intensity of about $1.1 \times 10^{21} \mathrm{~W} / \mathrm{cm}^{2}$, when the detection direction is perpendicular to the propagation directions of both the electron and laser beam. The effects of the carrierenvelop phase and the intensity of the laser pulse on the dynamics and radiation characteristics of the electron are also discussed.

\section{FORMULATION}

The interaction geometry is shown schematically in Fig. 1. The laser field is described by the normalized vector potential $\mathbf{a}=e \mathbf{A} / m_{e} c$, where $\mathbf{A}$ is the vector potential of the laser pulse, $m_{e}$ and $e$ are the electron mass and charge, respectively, and $c$ is the speed of light in vacuum. The normalized vector potential of an ultrashort laser pulse is expressed by

$$
\begin{aligned}
\mathbf{a}= & \frac{a_{0}}{\sqrt{2}} f(\eta)\left[\sqrt{1+\delta} \cos \left(\eta+\phi_{0}\right) \mathbf{e}_{x}\right. \\
& \left.+\sqrt{1-\delta} \sin \left(\eta+\phi_{0}\right) \mathbf{e}_{y}\right]
\end{aligned}
$$

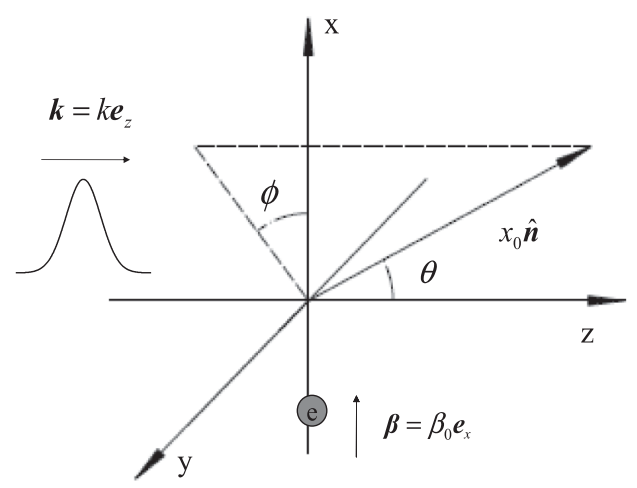

FIG. 1. Sketch of the nonlinear Thomson scattering. where $f(\eta)$ is the temporal envelop function of the laser pulse, $\phi_{0}$ is the phase difference between the maximum of the carrier envelop and the carrier. $\delta$ is the polarization parameter, $\delta= \pm 1$ for linear polarization and $\delta=0$ for circular polarization. Transverse homogeneous plane wave approximation is applied to the laser pulse so that it is a function of only the parameter $\eta=t-\hat{\mathbf{k}} \cdot \mathbf{r}$, where $\hat{\mathbf{k}}=\mathbf{k} /|\mathbf{k}|$. In the above expression, the space and time coordinates are normalized by $|\mathbf{k}|^{-1}$ and $\omega_{0}^{-1}$, respectively. $\mathbf{k}$ and $\omega_{0}$ are the wave vector and frequency of the laser pulse. The applicability of the plane wave approximation will be discussed later.

For a Gaussian laser pulse, the temporal shape function is written by

$$
f(\eta)=e^{-\left(\eta / \eta_{0}\right)^{2}},
$$

where $\eta_{0}$ is a parameter related to the pulse duration and is determined by the number of optical cycles $N$ within the carrier envelop by $\eta_{0}=N 2 \pi / \sqrt{2 \ln 2}$.

The motion of an electron in the laser field is governed by the following Lorentz equation:

$$
\frac{d(\gamma \boldsymbol{\beta})}{d t}=\frac{\partial \mathbf{a}}{\partial t}-\boldsymbol{\beta} \times(\boldsymbol{\nabla} \times \mathbf{a}),
$$

where $\boldsymbol{\beta}=\frac{\mathbf{v}}{c}$ is the normalized electron velocity and $\gamma=\frac{1}{\sqrt{1-\beta^{2}}}$ is the relativistic factor.

The explicit expressions for the trajectory and normalized velocity of the electron are given by [5]

$$
\begin{aligned}
\mathbf{r}(\eta)= & \mathbf{r}_{0}+\int \frac{\boldsymbol{\beta}_{0}+\frac{\mathbf{a}\left(\eta^{\prime}\right)}{\gamma_{0}}}{1-\hat{\mathbf{k}} \cdot \boldsymbol{\beta}_{0}} d \eta^{\prime} \\
& +\hat{\mathbf{k}} \int \frac{\frac{1}{2}\left(\frac{\mathbf{a}\left(\eta^{\prime}\right)}{\gamma_{0}}\right)^{2}+\frac{\mathbf{a}\left(\eta^{\prime}\right)}{\gamma_{0}} \cdot \boldsymbol{\beta}_{0}}{\left(1-\hat{\mathbf{k}} \cdot \boldsymbol{\beta}_{0}\right)^{2}} d \eta^{\prime}, \\
\boldsymbol{\beta}(\eta)= & \frac{\boldsymbol{\beta}_{0}+\frac{\mathbf{a}(\eta)}{\gamma_{0}}+\hat{\mathbf{k}} \frac{\left.\frac{1}{2} \frac{\left(\frac{a}{2}(\eta)\right.}{\gamma_{0}}\right)^{2}+\frac{\mathbf{a}(\eta)}{\gamma_{0}} \cdot \boldsymbol{\beta}_{0}}{1-\hat{\mathbf{k}} \cdot \boldsymbol{\beta}_{0}}}{1+\frac{\frac{1}{2}\left(\frac{\mathbf{a} \eta}{\gamma_{0}}\right)^{2}+\frac{\mathbf{a}(\eta)}{\gamma_{0}} \cdot \boldsymbol{\beta}_{0}}{1-\hat{\mathbf{k}} \cdot \boldsymbol{\beta}_{0}}}
\end{aligned}
$$

where $\mathbf{r}_{0}, \boldsymbol{\beta}_{0}$, and $\gamma_{0}$ are the initial position, velocity, and relativistic factor of the electron before the interaction.

After obtaining the orbit and velocity of the electron in the laser field by the above expressions, the radiation power per solid angle detected far away from the electron can be calculated by

$$
\begin{gathered}
\frac{d P(t)}{d \Omega}=|\mathbf{A}(t)|^{2}, \\
\mathbf{A}(t)=\sqrt{\frac{e^{2}}{16 \pi^{2} \varepsilon_{0} c}} \frac{\hat{\mathbf{n}} \times\left\{\left[\hat{\mathbf{n}}-\boldsymbol{\beta}\left(t^{\prime}\right)\right] \times \dot{\boldsymbol{\beta}}\left(t^{\prime}\right)\right\}}{\left[1-\hat{\mathbf{n}} \cdot \boldsymbol{\beta}\left(t^{\prime}\right)\right]^{3}},
\end{gathered}
$$

where $\varepsilon_{0}$ is the vacuum permittivity, $t^{\prime}$ is the retarded time when the electron radiates and relates to the time $t$ when the radiation arrives at the detector by 


$$
t=t^{\prime}+x_{0}-\hat{\mathbf{n}} \cdot \mathbf{r}\left(t^{\prime}\right)
$$

where $x_{0}$ is the distance from the origin to the detector, and $\hat{\mathbf{n}}=\sin \theta \cos \phi \mathbf{e}_{x}+\sin \theta \sin \phi \mathbf{e}_{y}+\cos \theta \mathbf{e}_{z}$ is the unit vector along the detection direction determined by the polar angle $\theta$ and azimuthal angle $\phi$ as shown in Fig. 1 .

The integrated energy emitted along the direction $\hat{\mathbf{n}}$ can be obtained by

$$
\frac{d W}{d \Omega}=\int_{-\infty}^{+\infty} \frac{d P(t)}{d \Omega} d t
$$

Further, the spectral intensity per solid angle of the radiation emitted by the electron along the direction $\hat{\mathbf{n}}$ can be calculated by

$$
\frac{d^{2} I}{d \omega d \Omega}=\frac{e^{2} \omega^{2}}{16 \pi^{3} \varepsilon_{0} c}\left|\int_{-\infty}^{+\infty} d t[\hat{\mathbf{n}} \times(\hat{\mathbf{n}} \times \boldsymbol{\beta})] e^{i \omega(t-\hat{\mathbf{n}} \cdot \mathbf{r})}\right|^{2} .
$$

\section{RESULTS AND DISCUSSION}

The radiation characteristics of nonlinear Thomson scattering with various laser and electron parameters are investigated. Figure 2(a) shows the time history of the radiation power per solid angle with the following parameters: the circularly polarized laser pulse propagates along the $+z$ axis with the carrier-envelop phase $\phi_{0}=\frac{3}{4} \pi$, wavelength $\lambda_{0}=800 \mathrm{~nm}, N=3$ corresponding to the pulse duration of 8 femtosecond, and $a_{0}=19.57$ corresponding to the intensity of $1.1 \times 10^{21} \mathrm{~W} / \mathrm{cm}^{2}$, the electron with an initial energy of $5 \mathrm{MeV}$ moves along the $+x$ axis, the radiation is detected along $+y$ axis, which is perpendicular to the propagation directions of both the laser pulse and the electron. The peak is magnified as shown in Fig. 2(b) to look at the pulse shape in detail. It is clear to see that a single ultrashort pulse is generated. The angular spectral intensity of the radiation is plotted in Fig. 3. The spectrum peaks at about $1.3 \mathrm{keV}$ and has a high energy tail extending beyond $10 \mathrm{keV}$ which supports the ultrashort pulse as in Fig. 2.

Usually, the $90^{\circ}$ Thomson scattering is performed with a linearly polarized laser pulse and the radiation is detected along the electron propagation direction. For comparison, the time history of the radiation power per solid angle is shown in Fig. 4 by changing the laser pulse to be linearly polarized and the radiation detection direction to be along the $+x$ axis while all of the other parameters are kept the same as those in Fig. 2. A train of ultrashort pulses is obtained. The highest peak is magnified in Fig. 4(b) to show the pulse shape in detail. The maximum radiation power is higher and the pulse duration is shorter for a linearly polarized laser pulse because the peak value of a is $\sqrt{2}$ times as large as that of a circularly polarized pulse with the same value of $a_{0}$. The angular spectral intensity of the radiation is plotted in Fig. 5. Modulations of the
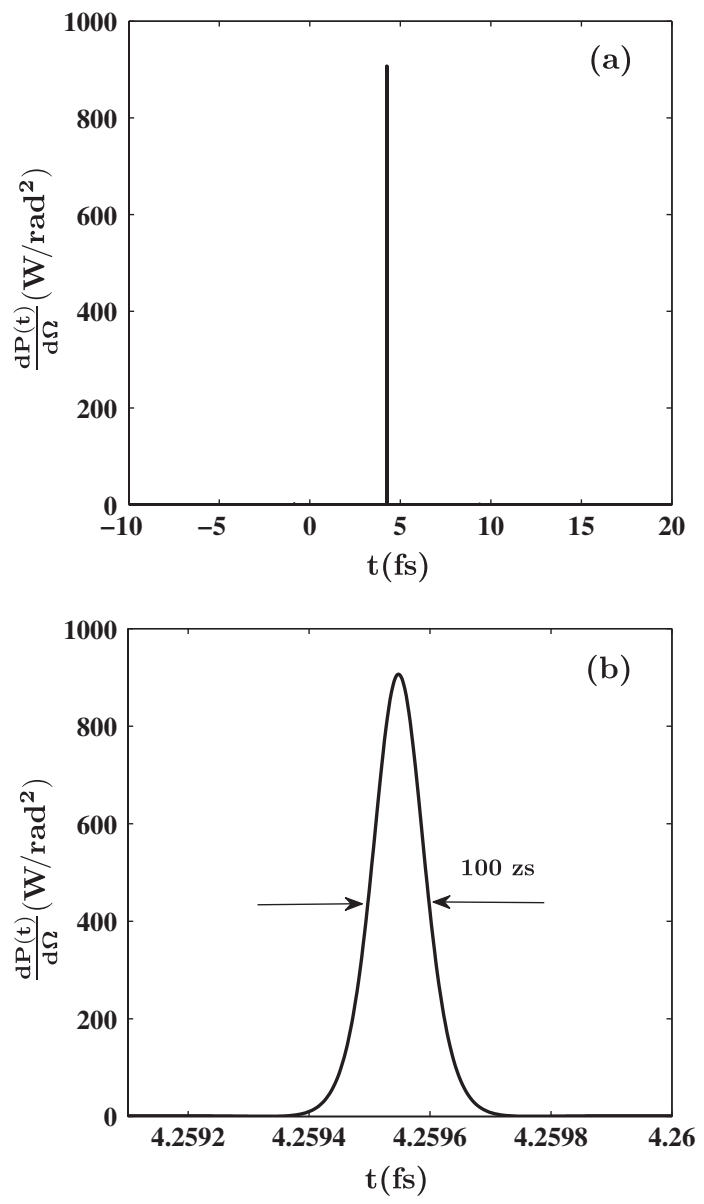

FIG. 2. (a) The time histogram of the radiation power per solid angle along the $+y$ axis by an electron scattering with a circularly polarized laser pulse. (b) The peak is magnified to show the details of the pulse shape. The parameters taken in the calculations are as follows: the laser pulse propagates along the $+z$ axis with $a_{0}=19.57, N=3, \phi_{0}=\frac{3}{4} \pi, \lambda_{0}=800 \mathrm{~nm}$ and $\delta=0$, the electron with an initial energy of $5 \mathrm{MeV}$ moves along the $+x$ axis, and the detection direction $\hat{\mathbf{n}}=\mathbf{e}_{y}$.

spectrum can be seen which is due to the slight differences of the intervals between the peaks in Fig. 4(a).

In order to get more insight into the underlying mechanism for the generation of a single ultrashort pulse by nonlinear Thomson scattering of a circularly polarized laser pulse, the normalized velocity of the electron as a function of $\eta$ during the interaction process is plotted in Fig. 6. We can see that $\beta_{x}$ is close to 1 before the interaction, starts to decrease and oscillate at the front edge of the laser pulse while $\beta_{y}$ and $\beta_{z}$ start to oscillate too and the oscillation amplitude increases. $\beta_{y}$ reaches the maximum close to 1 at $\eta=0$. There is a factor of $\left[1-\hat{\mathbf{n}} \cdot \boldsymbol{\beta}\left(t^{\prime}\right)\right]^{-6}$ included in the expressions of the radiation power indicated in Eqs. (6) and (7). During the acceleration process, the radiation power can be significantly enhanced if the normalized electron velocity along the detection direction approaches 1 , as at the time $\eta=0$ in our case. The 


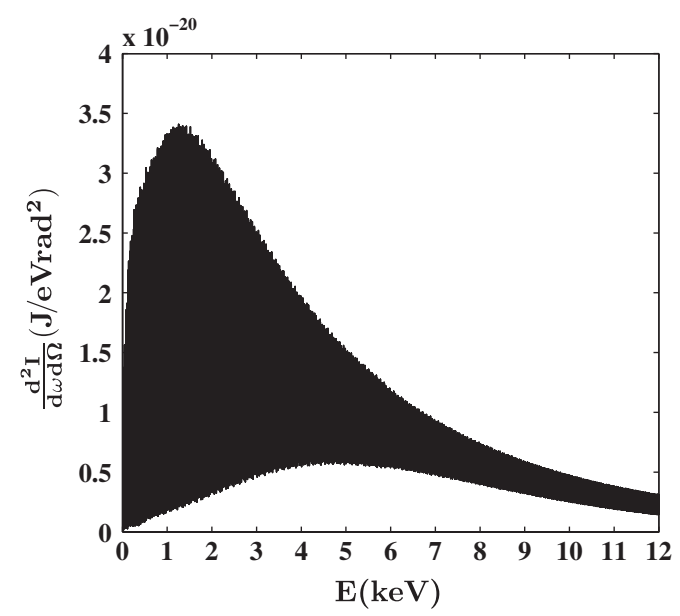

FIG. 3. The spectral intensity per solid angle along the $+y$ axis radiated by an electron scattering with a circularly polarized laser pulse. All of the calculation parameters are the same as those in Fig. 2.
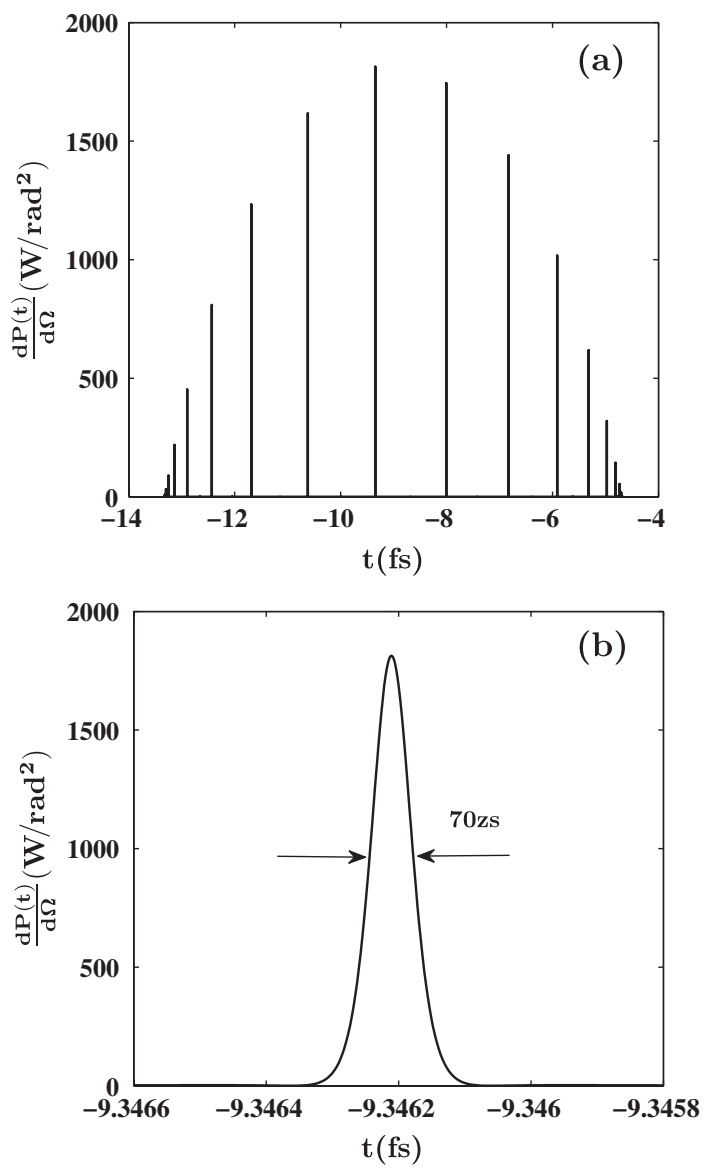

FIG. 4. (a) The time histogram of the radiation power per solid angle along the $+x$ axis by an electron scattering with a linearly polarized laser pulse. (b) The highest peak is magnified to show the details of the pulse shape. All of the calculation parameters are the same as those in Fig. 2 except that $\delta=1$ and $\hat{\mathbf{n}}=\mathbf{e}_{x}$.

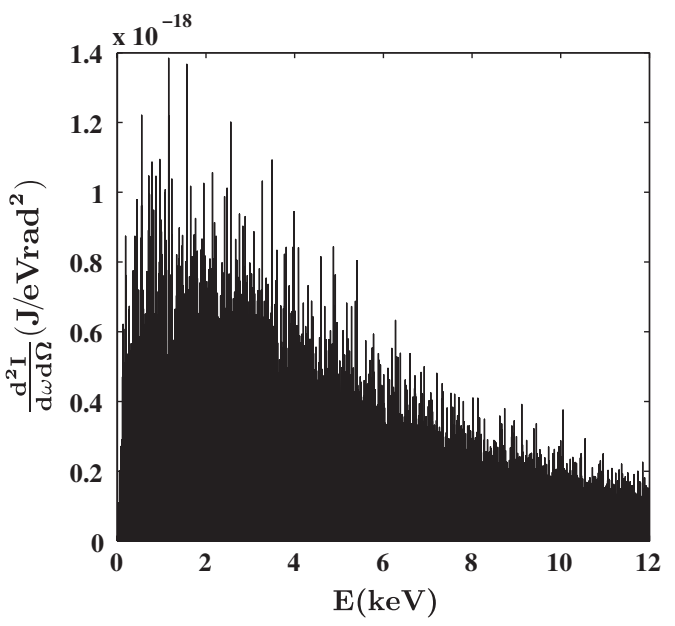

FIG. 5. The spectral intensity per solid angle along the $+x$ axis radiated by an electron scattering with a linearly polarized laser pulse. All of the calculation parameters are the same as those in Fig. 4.

radiation at this time is much stronger than that any other time resulting in the generation of an ultrashort pulse as shown in Fig. 2(a).

Substituting $\delta=0$ in Eq. (1), $\boldsymbol{\beta}_{0}=\beta_{0} \mathbf{e}_{x}$ and $\hat{\mathbf{k}}=\mathbf{e}_{z}$ in Eq. (5), we can obtain the expression for $\beta_{y}$ :

$\beta_{y}(\eta)=\frac{\frac{1}{\sqrt{2}} \frac{a_{0}}{\gamma_{0}} \beta_{0} e^{-\left(\eta / \eta_{0}\right)^{2}} \sin \left(\eta+\phi_{0}\right)}{1+\frac{1}{4}\left(\frac{a_{0}}{\gamma_{0}}\right)^{2} e^{-2\left(\eta / \eta_{0}\right)^{2}}+\frac{1}{\sqrt{2}} \frac{a_{0}}{\gamma_{0}} \beta_{0} e^{-\left(\eta / \eta_{0}\right)^{2}} \cos \left(\eta+\phi_{0}\right)}$.

$\beta_{y}$ reaches the maximum when $\frac{\partial \beta_{y}}{\partial \eta}=0$ and $\frac{\partial \beta_{y}}{\partial a_{0}}=0$ while $\beta_{0}$ is given at a fixed value. After some straightforward algebra, these two equations give

$$
\cos \left(\eta+\phi_{0}\right)=-\frac{\beta_{0}}{\sqrt{2}}
$$

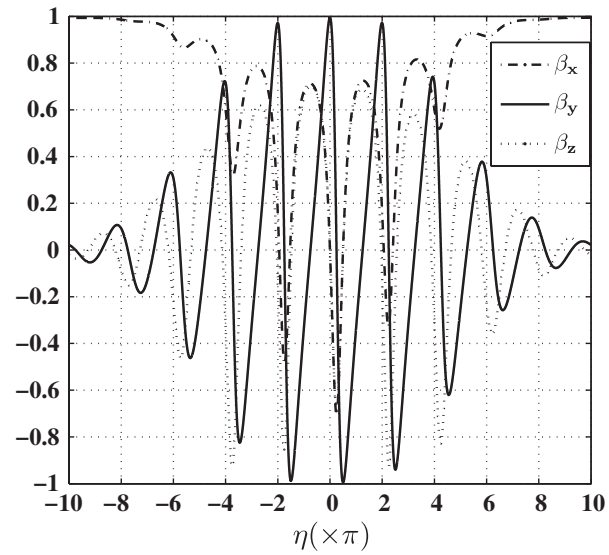

FIG. 6. The normalized velocity of the electron as a function of $\eta$ in a circularly polarized laser field. $\beta_{x}, \beta_{y}$, and $\beta_{z}$ are the projections of $\boldsymbol{\beta}$ on each of the coordinate axes. All of the calculation parameters are the same as those in Fig. 2. 


$$
\frac{a_{0}}{\gamma_{0}}=2 e^{\left(\eta / \eta_{0}\right)^{2}}
$$

For a relativistic electron, the initial velocity of the electron is very close to the speed of light so that the right-hand side of Eq. (12) can be replaced by $-\frac{1}{\sqrt{2}}$. We can obtain

$$
\eta_{ \pm}= \pm \frac{3}{4} \pi-\phi_{0}+2 n \pi
$$

where $n$ is an integer. $\beta_{y}$ reaches the maximum along the $+y$ axis and the $-y$ axis at the time $\eta_{+}$and $\eta_{-}$, respectively. By substituting the $\eta_{+}$into Eq. (13), we can obtain the corresponding laser intensity. The lowest possible optimal intensity for the generation of a single ultrashort pulse is $a_{0}=2 \gamma_{0}=19.57$ by letting $\eta_{+}=0$ in Eq. (14) when $\phi_{0}=\frac{3}{4} \pi$ and $n=0$, which are the parameters taken for the calculations shown in Fig. 2.

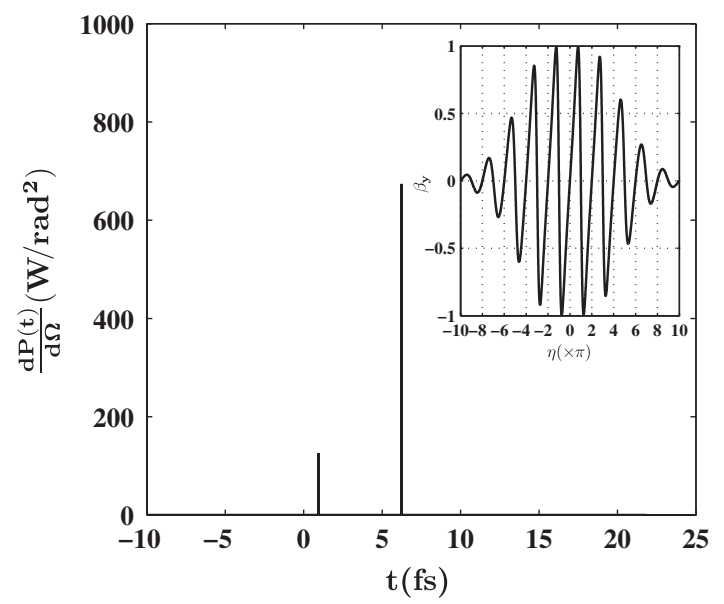

(a)

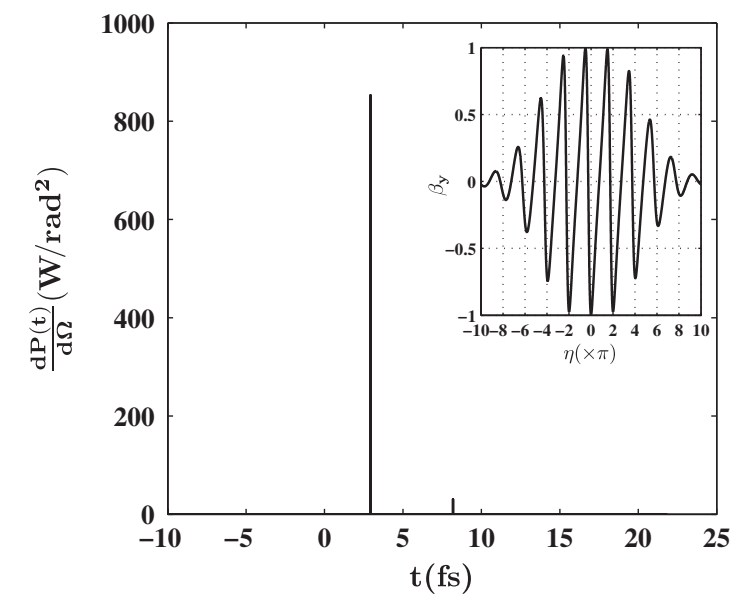

(c)
The dependences of the radiation characteristics on the carrier-envelop phase $\phi_{0}$ and the normalized vector potential $a_{0}$ of the laser pulse are investigated and shown in Figs. 7 and 8, respectively. Figure 7 shows the time history plots of radiation power per solid angle along the $+y$ axis by an electron in the laser fields with carrier-envelop phases of $\phi_{0}=0, \frac{1}{4} \pi, \frac{5}{4} \pi$, and $\frac{7}{4} \pi$. As shown in the inset of Fig. 7(a), when $\phi_{0}=0, \beta_{y}$ reaches the maximum close to 1 at $\eta_{+}=\frac{3}{4} \pi$ and the other time when $\beta_{y}$ gets close to 1 locates at $\eta=\frac{3}{4} \pi-2 \pi$. As discussed before, the radiation power is significantly enhanced when the speed is approaching 1, which results in the generation of the two pulses in Fig. 7(a). When $\phi_{0}=\frac{1}{4} \pi, \beta_{y}$ reaches the maximum at $\eta_{+}=\frac{1}{2} \pi$ which gets closer to the time when the laser intensity reaches its peak value at $\eta=0$. The other peak of $\beta_{y}$ locates at $\eta=\frac{1}{2} \pi-2 \pi$ which gets farther away from $\eta=0$. The slight change of $\beta_{y}$ causes the increase of the radiation power of the high pulse and the

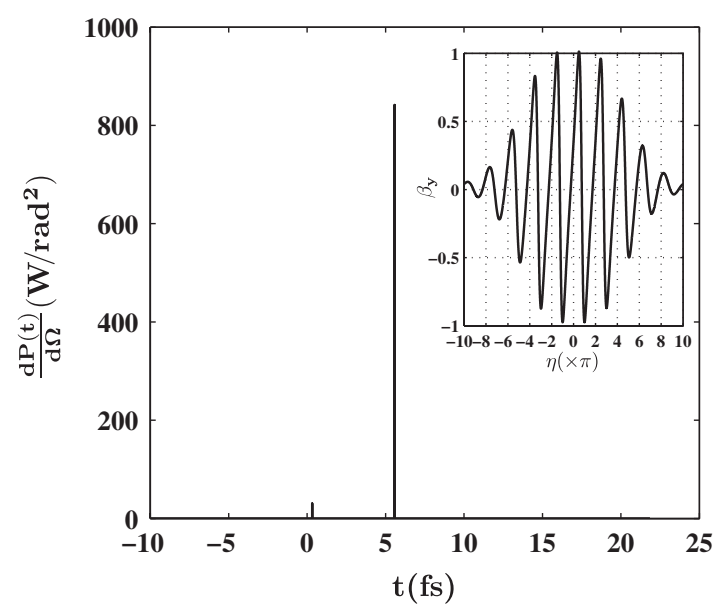

(b)

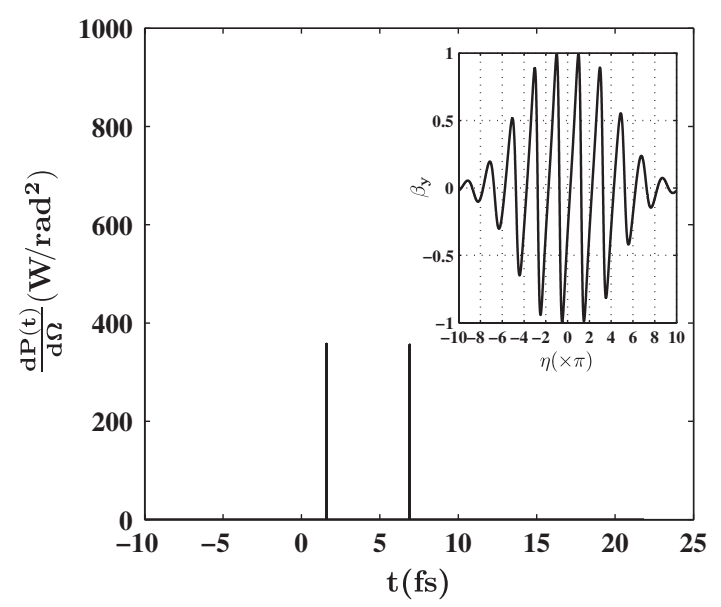

(d)

FIG. 7. The time histogram of the radiation power per solid angle along the $+y$ axis by an electron scattering with a circularly polarized laser pulse. The insets show the normalized velocity $\beta_{y}$ of the electron in the laser field as a function of $\eta$. (a) $\phi_{0}=0$, (b) $\phi_{0}=\frac{1}{4} \pi$, (c) $\phi_{0}=\frac{5}{4} \pi$, (d) $\phi_{0}=\frac{7}{4} \pi$. All of the other parameters are the same as those in Fig. 2 . 
decrease of the low pulse as shown in Fig. 7(b). By this effect, only a single pulse can be seen when $\phi_{0}=\frac{3}{4} \pi$ as shown in Fig. 2. A small pulse appears again in Fig. 7(c) when $\phi_{0}=$ $\frac{5}{4} \pi$ because $\beta_{y}$ gets close to 1 at $\eta=-\frac{1}{2} \pi+2 \pi$ as shown in the inset of Fig. 7(c). When $\phi_{0}$ is further increased to $\frac{7}{4} \pi$, the two peaks of $\beta_{y}$ locating at $\eta= \pm \pi$ have the same distances to $\eta=0$, so that twin radiation pulses have the same heights are generated as shown in Fig. 7(d).

Figure 8 shows the time history plots of radiation power per solid angle along the $+y$ axis by an electron in the laser fields with an intensity of $a_{0}=15,18,21$, and 22.83 . When the laser intensity $a_{0}<2 \gamma_{0}=19.57$, the electron oscillating velocity $\beta_{y}$ reaches its maximum at $\eta=0$ as shown in the inset of Fig. 8(a). The radiation power at this moment is stronger than that at any other time, resulting in the generation of the highest pulse in Fig. 8(a). Two other pulses with much lower power can also be seen due to the two peaks of $\beta_{y}$ at $\eta= \pm 2 \pi$. When the laser intensity increases to $a_{0}=18$, the maximum of $\beta_{y}$ gets close to 1 , a single pulse can be seen as in Fig. 8(b). When the laser intensity is at the lowest optimal value of $a_{0}=19.57$, a single pulse with much higher power is generated as shown in Fig. 2(a). When the laser intensity increases further to $a_{0}=21$, the peaks of $\beta_{y}$ at $\eta= \pm 2 \pi$ are also close to 1 and three pulses are generated as seen in Fig. 8(c). When the laser intensity is at the second optimal value of $a_{0}=$ 22.83 by letting $n=1$ in Eq. (14), $\beta_{y}$ reaches its maximum at $\eta= \pm 2 \pi$ as shown in Fig. 8(d), and two pulses with the same power as that in Fig. 2(a) are generated.

All of the above calculations are based on transverse plane wave approximation. This assumption is valid only when the transverse and longitudinal displacements of the electron during the interaction process are much less than the focal spot size $W_{0}$ and Rayleigh length $Z_{R}$ of the laser pulse, respectively. The trajectory of the electron during the scattering process is shown in Fig. 9 while all of the

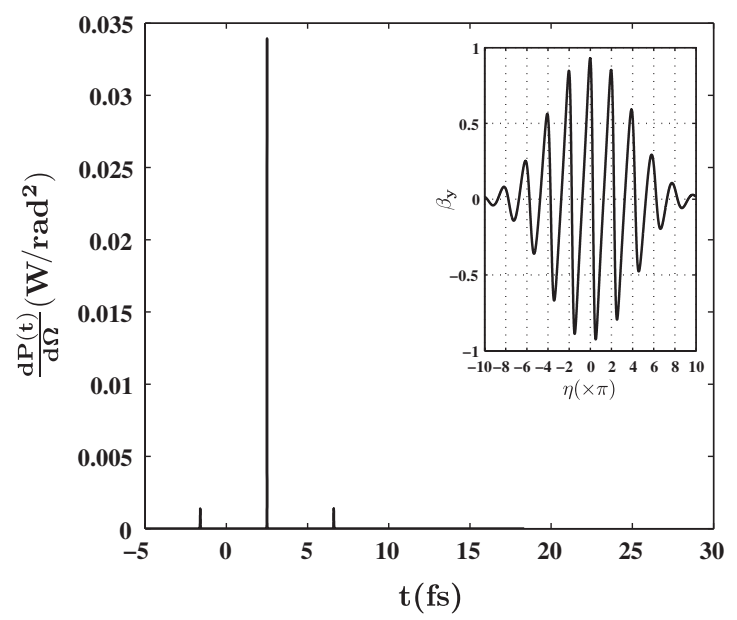

(a)

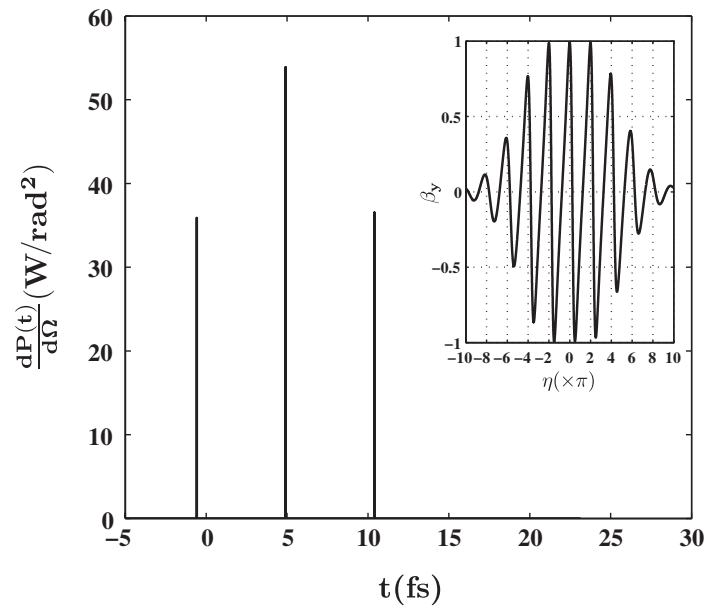

(c)

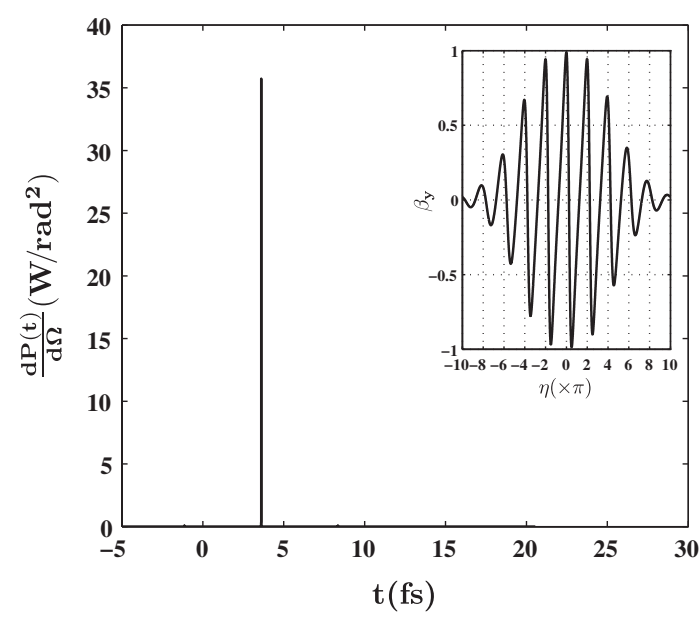

(b)

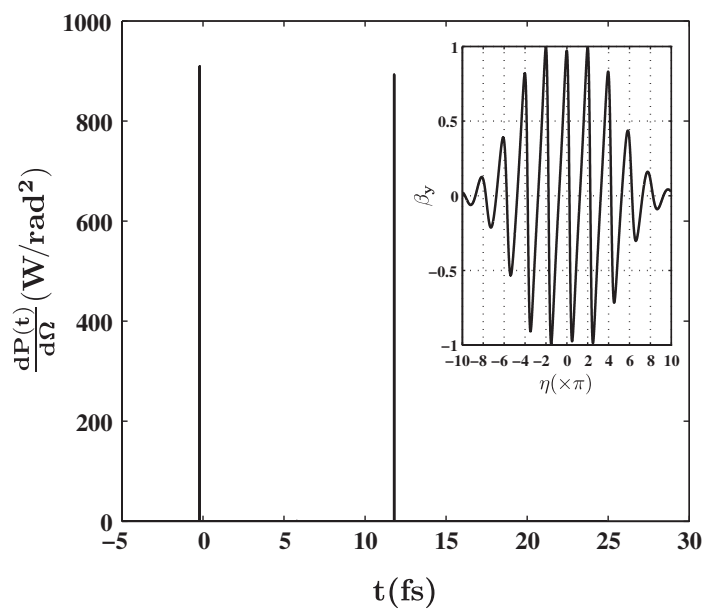

(d)

FIG. 8. The time histogram of the radiation power per solid angle along the $+y$ axis by an electron scattering with a circularly polarized laser pulse. The insets show the normalized velocity $\beta_{y}$ of the electron in the laser field as a function of $\eta$. (a) $a_{0}=15$, (b) $a_{0}=18$, (c) $a_{0}=21$, (d) $a_{0}=22.83$. All of the other parameters are the same as those in Fig. 2. 
calculation parameters are kept the same as those in Fig. 2. The displacement along the $y$ axis $r_{y}$ is less than $0.2 \mu \mathrm{m}$ and the longitudinal displacement along the $z$ axis $r_{z}$ is less than $3 \mu \mathrm{m} . r_{y} \ll W_{0}$ and $r_{z} \ll Z_{R}$ is usually valid because in practical experiment, the focal spot size of the laser beam is a few ten micrometers and the corresponding Rayleigh length is hundreds of micrometers. The displacement $r_{x}$ is much larger than $r_{y}$ due to the fact that the initial velocity of the electron is along the $+x$ axis. According to the results in Figs. 2 and 6, and the discussions made before, most of the radiation is produced during the three optical cycles close to the peak of the laser pulse. We estimate the interaction time by $\Delta t=N \frac{\lambda_{0}}{c}$ which is taken by the laser pulse to propagate across the electron. The displacement of the electron along the $+x$ axis during this time is given by $\Delta x=\int_{0}^{\Delta t} c \beta_{x} d t \leq c \Delta t=N \lambda_{0}$. The condition $\Delta x \ll W_{0}$ is satisfied if $N \lambda_{0} \ll W_{0}$. Taking the values $N=3, \lambda_{0}=0.8 \mu \mathrm{m}, I=1.1 \times 10^{21} \mathrm{~W} / \mathrm{cm}^{2}$ and assuming $W_{0}=10 N \lambda_{0}$, it can be estimated that a laser system with peak power in the petawatt $\left(10^{15} \mathrm{~W}\right)$ range is needed for the experiment. It is possible to construct such a laser system with the state-of-the-art laser technology in the near future. For example, the petawatt field synthesizer, currently under construction at the Max-Planck-Institut für Quantenoptik (Garching, Germany) [22] is a project for developing a laser facility which is expected to deliver carrier-envelop phase-controlled laser pulses with petawatt-scale peak power in the few-cycle regime.

Finally, it needs to emphasize that the laser pulse always interacts with a group of electrons in real experiment. The radiations from different electrons would spread in time due to the different interaction time. In order to keep the extremely short pulse duration when a bunch of electrons is involved, the radiations should be added coherently. The condition for coherent radiations of a group of electrons is discussed by Lee et al. [23]. The pulse duration of the radiation of an electron bunch is the same as that of a single electron when a laser pulse interacts with an ultrathin target which is placed to let

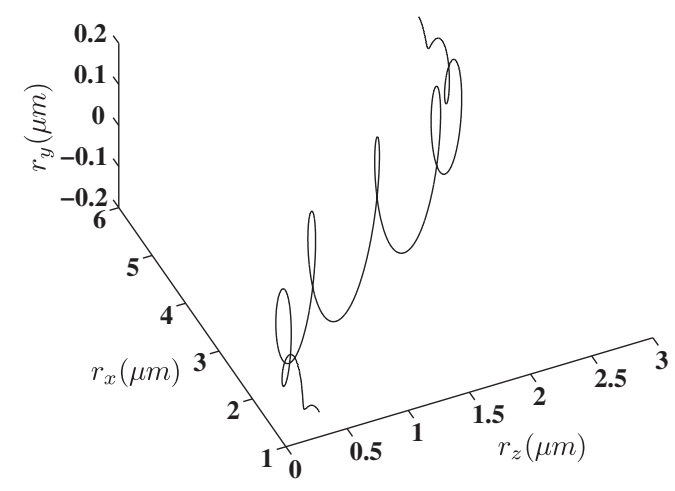

FIG. 9. The electron trajectory during the interaction. All of the calculation parameters are the same as those in Fig. 2.

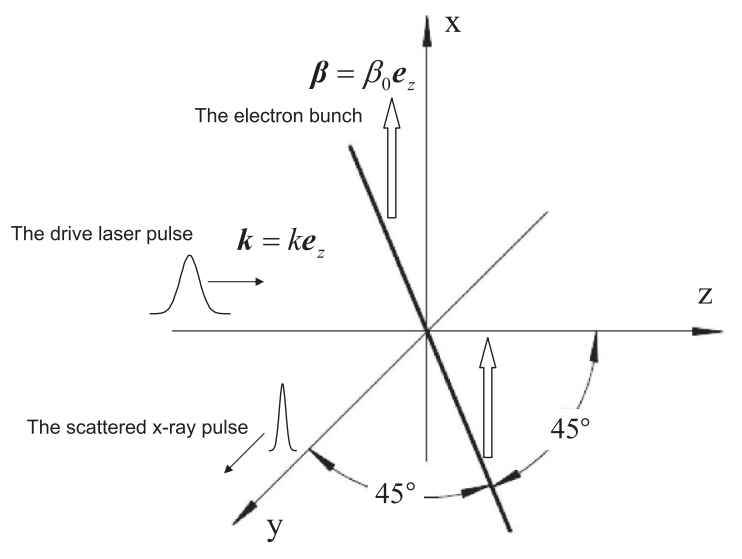

FIG. 10. Sketch of the interaction geometry in order to keep the ultrashort pulse duration of the radiations when an electron bunch is involved.

the reflection direction of the incident laser coincide with the radiation direction. Considering our interaction geometry, this condition could be realized by a slice of electrons moving along the $+x$ axis and having the same angle of $45^{\circ}$ with respect to the $y$ and $z$ axes as shown schematically in Fig. 10. Such a bunch of electrons is quite difficult to be achieved with conventional accelerators. However, it could be produced by the acceleration of the electrons out of a nanowire using another intense laser pulse as studied by Wu et al. [24,25].

\section{SUMMARY}

In conclusion, the dynamics and radiation characteristics of a relativistic electron in the intense laser field are investigated numerically. The results show that an ultrashort $\mathrm{x}$-ray pulse can be generated by an electron with an initial energy of $5 \mathrm{MeV}$ propagating across a circularly polarized laser pulse with a duration of 8 femtoseconds and an intensity of about $1.1 \times 10^{21} \mathrm{~W} / \mathrm{cm}^{2}$, when the detection direction is perpendicular to the propagation directions of both the electron and the laser beam. When the normalized velocity of the electron along the detection direction is approaching 1, the radiation power is significantly enhanced and much stronger than the radiation power at any other time so that a single ultrashort pulse is generated. The influence of the carrier-envelop phase $\phi_{0}$ and the normalized intensity $a_{0}$ of the laser pulse on the motion and the radiation characteristics of the electron is discussed. It is found that a single ultrashort pulse is efficiently generated by an electron with relativistic factor of $\gamma_{0}$ scattering with a circularly polarized laser pulse with $\phi_{0}=\frac{3}{4} \pi$ and $a_{0}=2 \gamma_{0}$.

\section{ACKNOWLEDGMENTS}

This work is supported by DFG Transregio SFB/TR18 and GRK 1203 programs. 
[1] J.J. Thomson, The Corpuscular Theory of Matter (A. Constable \& Co., Ltd., London, 1907).

[2] D. Strickland and G. Mourou, Opt. Commun. 56, 219 (1985).

[3] Vachaspati, Phys. Rev. 128, 664 (1962).

[4] E. S. Sarachik and G. T. Schappert, Phys. Rev. D 1, 2738 (1970).

[5] Y. I. Salamin and F. H. M. Faisal, Phys. Rev. A 54, 4383 (1996).

[6] E. Esarey, S. K. Ride, and P. Sprangle, Phys. Rev. E 48, 3003 (1993).

[7] S. K. Ride, E. Esarey, and M. Baine, Phys. Rev. E 52, 5425 (1995).

[8] B.F. Shen, W. Yu, G. H. Zeng, and Z.Z. Xu, Opt. Commun. 136, 239 (1997).

[9] W. Yu, M. Y. Yu, J. X. Ma, and Z. Xu, Phys. Plasmas 5, 406 (1998).

[10] G. A. Krafft, Phys. Rev. Lett. 92, 204802 (2004).

[11] J. Gao, Phys. Rev. Lett. 93, 243001 (2004).

[12] A. Popa, Phys. Rev. A 84, 023824 (2011).

[13] S. Y. Chen, A. Maksimchuk, and D. Umstadter, Nature (London) 396, 653 (1998).

[14] S. Banerjee, A. R. Valenzuela, R. C. Shah, A. Maksimchuk, and D. Umstadter, Phys. Plasmas 9, 2393 (2002).

[15] K. T. Phuoc, A. Rousse, M. Pittman, J. P. Rousseau, V. Malka, S. Fritzler, D. Umstadter, and D. Hulin, Phys. Rev. Lett. 91, 195001 (2003).
[16] K. Lee, Y.H. Cha, M.S. Shin, B.H. Kim, and D. Kim, Phys. Rev. E 67, 026502 (2003).

[17] F. Krausz and M. Ivanov, Rev. Mod. Phys. 81, 163 (2009).

[18] P. Zhang, Y. R. Song, and Z. G. Zhang, Phys. Rev. A 78, 013811 (2008).

[19] P. F. Lan, P. X. Lu, and W. Cao, Phys. Plasmas 13, 013106 (2006).

[20] P. F. Lan, P. X. Lu, W. Cao, and X. L. Wang, Phys. Rev. E 72, 066501 (2005).

[21] L. Liu, C. Q. Xia, J. S. Liu, W. T. Wang, Y. Cai, C. Wang, R.X. Li, and Z.Z. Xu, Laser Part. Beams 28, 27 (2010).

[22] Z. Major, S. A. Trushin, I. Ahmad, M. Siebold, C. Wandt, S. Klingebiel, T. J. Wang, J. A. Fülöp, A. Henig, S. Kruber, R. Weingartner, A. Popp, J. Osterhoff, R. Hörlein, J. Hein, V. Pervak, A. Apolonski, F. Krausz, and S. Karsch, Rev. Laser Eng. 37, 431 (2009) [http:// www.attoworld.de/Documents/papers/LaserReview/ LaserReview37_6p431_2009.pdf].

[23] K. Lee, B. H. Kim, and D. Kim, Phys. Plasmas 12, 043107 (2005).

[24] H.C. Wu, J. Meyer-ter-Vehn, J. Fernandez, and B. M. Hegelich, Phys. Rev. Lett. 104, 234801 (2010).

[25] H. C. Wu, Appl. Phys. Lett. 99, 021503 (2011). 